\title{
APRENDIZAJE DE ACTIVIDADES MOTORAS EN PROCESOS PRENSILES CON EL USO DE UNA PRÓTESIS MECATRÓNICA CONTROLADA POR SEÑALES MIOELÉCTRICAS EN PERSONAS AMPUTADAS ENTRE CODO Y MUÑECA
}

rev.ciencia.poder.aéreo.6: 31 - 35, 2011.

\author{
Autor ${ }^{1}$ \\ John Jairo Páez Rodríguez \\ Fecha recepción: 29 de marzo de 2011. \\ Fecha aceptación: 27 de Junio de 2011.
}

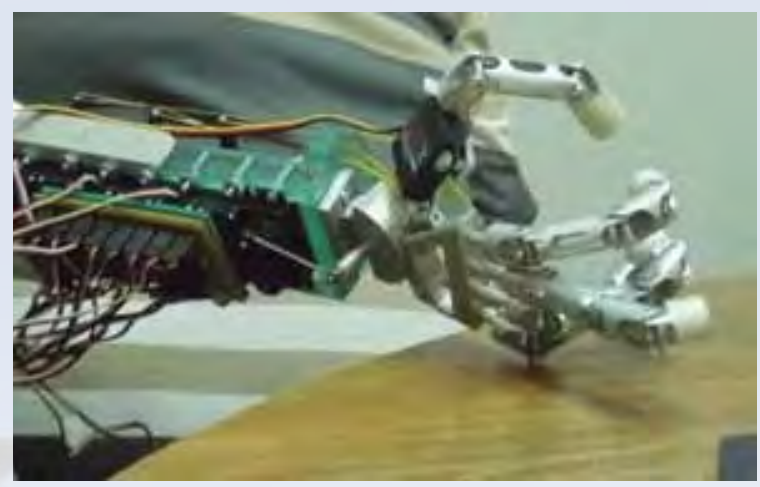

Resumen

El proyecto de investigación en el campo de las ayudas aumentativas para discapacitados, tiene como objetivo principal desarrollar una prótesis del sistema biomecánico mano-muñeca para investigar la influencia que tiene en el desarrollo de ciertas habilidades y configuraciones cerebrales durante el proceso de aprendizaje; dado que la mano: permite crear un sistema de autorrepresentación, es un punto de convergencia en el sistema de retroalimentación visual, táctil y propioceptiva (percepción de la propia posición), materializa las intenciones del pensamiento, al tener conexión con el sistema ventral tiene acceso a las áreas lingüísticas de los lóbulos temporales donde se desarrollan los procesos semánticos (debido a la relación de manipulación -identificación- transformación de objetos), ofrece al cerebro nuevos modos de aproximarse a viejas tareas y posibilidad de emprender otras nuevas $y$, porque el cerebro a través de la mano modela su proceso, formación del mundo y su espacio extrapersonal.

Palabras clave: Aprendizaje de rutinas motoras, señales mioeléctricas, ambiente de aprendizaje por observación, ambiente de aprendizaje somatognósico, prótesis mecatrónica.

\section{Abstract}

This project makes part of an investigation about augmentative aids for disabled people, and has as main objective to develop a prosthesis of the biomechanical system hand - wrist in order to investigate the influence that it has in the development of certain abilities and cerebral configurations during the process of learning.

Due to the hand permits to create a system of self-representation, it is a point of convergence in the system of visual, tactile and proprioceptive (perception of the own position) feedback. Besides this, it materializes

${ }^{1}$ Licenciado en Diseño Tecnológico - Universidad Pedagógica Nacional. Magister en Tecnologías de la Información Aplicadas a la Educación - Universidad Pedagógica Nacional. Magister(c) en Ingeniería de Sistemas y Computación - Pontificia Universidad Javeriana. Investigador Principal Grupo SynapsisUPN - Universidad Pedagógica Nacional. Correo electrónico: john_jpr@hotmail.com - jjpaerzr@udistrital.edu.co 


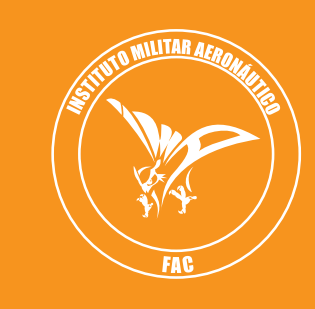

Tecnología

Revista Ciencia y Poder Aéreo. Edición No 6 - Septiembre 2011 ISSN 1909 - 7050

the intentions of the thought through the connection with the ventral system accessing the linguistic areas of the temporary lobes where semantic processes are developed (due to the relation of handling - identification - transformation of objects).

In the same way, it offers the brain new ways of approaching old tasks and the possibility of undertaking new ones and, because the brain through the hand models its process, makes the world and its extra personal space.

Key words: Learning of motor routines, myoelectric signals, observation learning environment, somatotype learning environment, mechatronical prosthesis.

\section{Introducción}

El proyecto de investigación en el campo de las ayudas aumentativas para discapacitados, tiene como objetivo principal evaluar la interacción de una prótesis mecatrónica controlada por señales mioeléctricas emitidas por una persona con amputación traumática a nivel del tercio distal del codo que le permita aprender a realizar de forma más eficaz la actividad motora de procesos prensiles de precisión. Esto es de gran importancia a nivel cognitivo ya que la mano permite crear un sistema de auto representación, es un punto de convergencia en el sistema de retroalimentación visual y propioceptiva (percep-
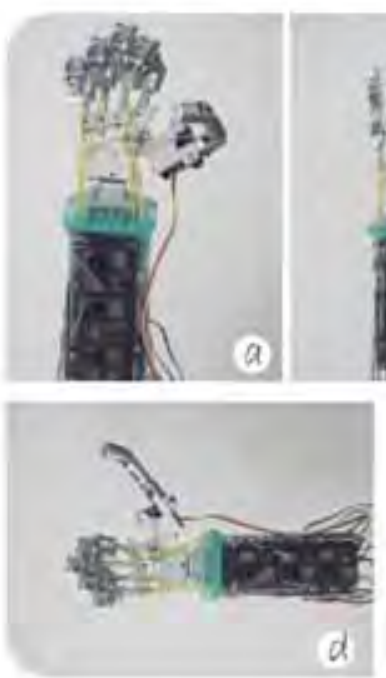

Figura1. Prótesis mecatrónica desarrollada. ción de la propia posición), debido a la relación de manipulación -identificación- transformación de objetos, pues ofrece al cerebro nuevos modos de aproximarse a tareas anteriores y la posibilidad de emprender otras nuevas (Ver figura 1).

\section{Problema}

¿Una persona con amputación traumática de miembro superior a nivel del tercio distal del codo aprende rutinas motoras para procesos prensiles de precisión de forma más eficaz. Utilizando un ambiente de aprendizaje con la perspectiva de jerarquía de desplazamientos (ambiente por observación) ó utilizando un ambiente de aprendizaje con la perspectiva de desarrollo somatognósico?

\section{Objetivo General}

Evaluar la interacción de una prótesis mecatrónica controlada por señales mioeléctricas emitidas por una persona con amputación traumática a nivel del tercio distal del codo que le permita aprender a realizar de forma más eficaz la actividad motora de cinco procesos prensiles de precisión.

\section{Objetivos \\ Específicos}

Diseñar y construir una mano protésica que le permita a una persona discapacitada con amputación traumática a nivel entre el codo y la muñeca poder realizar procesos prensiles de precisión tomando señales mioeléctricas del propio cuerpo.

Tecnología. Aprendizaje de Actividades Motoras en Procesos Prensiles con el Uso de una Prótesis Mecatrónica Controlada por Señales Mioeléctricas en Personas Amputadas Entre Codo y Muñeca - John Jairo Páez Rodríguez 
Construir dos modelos de entrenamiento para la apropiación de la prótesis por parte del sujeto discapacitado.

Evaluar el proceso de aprendizaje y apropiación de la prótesis para cada uno de los modelos propuestos en términos del número de intentos de prensión (exitosos y no exitosos) para resolver las tareas propuestas de actividades motoras de prensión.

Identificar las estrategias cognitivas utilizadas por el sujeto discapacitado cuando resuelve los problemas de prensión utilizando la prótesis propuesta.

\section{Metodología para el desarrollo de la investigación}

Para el desarrollo de la investigación, se cuenta con dos sujetos mayores de edad, género masculino, con discapacidad de la ausencia de uno o ambos miembros superiores a la altura del tercio proximal del codo; la diagnosis que presentan se describe en términos de movilidad parcial del codo, funciones mentales globales y específicas normales, funciones sensoriales, de voz y habla normales.

Se han considerado como variables no intervinientes el performance, la conciencia corporal, praxia global con la prótesis y la memoria motriz. También, se considera la relación entre la conciencia corporal y la praxia en los procesos de prensión con la prótesis y sus dinámicas de funcionamiento en las personas que presentan limitación por la ausencia del miembro superior, ya que tanto la praxia global como la conciencia corporal presentan un desarrollo diferente y más lento por la falta de práctica o familiaridad con el sistema mecatrónico.

El desarrollo de los ambientes tiene un carácter evolutivo ya que los objetivos apuntan básicamente al aprendizaje de rutinas motoras y al mejoramiento de la praxia de la prótesis. A cada sujeto se le entrena en uno de los dos ambientes de aprendizaje con sus respectivas actividades $\mathrm{y}$, finalmente a los dos sujetos se les plantea ocho situaciones problema de manipulación de objetos para medir la eficiencia y eficacia y para definir la estrategia cognitiva utilizada por cada uno de ellos. Esto se desarrollará a través de la metodología de protocolos verbales desarrollada por Maldonado L. F. (2001), ya que esta permite evidenciar los procesos de razonamiento de cada sujeto cuando soluciona problemas de prensión con la prótesis y el desarrollo de las habilidades motoras relacionadas con el tono muscular de donde se obtiene la señal mioeléctrica que controla el sistema mecatrónico. En otras palabras, se estudiará la conciencia de los sujetos en la solución de problemas de prensión con el dispositivo mecatrónico mediante la introspección.

El inicio de este proceso requiere comprobar tres elementos básicos:

En la entrada de información se busca un conocimiento exacto y preciso de la información necesaria para desarrollar las actividades de prensión en cualquiera de los dos ambientes. Una estrategia es presentar al sujeto los parámetros de la tarea con la mayor simplicidad y familiaridad, evitando así, un conocimiento impreciso de la tarea a desarrollar.

Previo al proceso de elaboración de la rutina motora es necesario conocer en el sujeto la percepción y definición del problema y así comprobar si la delimitación del problema de prensión con el miembro superior es clara. Aquí es necesario conocer de forma verbal la conducta de planificación que establece un plan que se quiere utilizar dada la información adquirida previamente para alcanzar la meta. En este sentido, se tiene en cuenta que el sujeto establezca metas claras y sea consciente de las mismas, ya que estas metas se consideran responsables de la búsqueda del significado de la tarea y además se crea la necesidad de ordenar, organizar y estructurar todos los pasos o momentos del proceso para terminar con éxito la tarea de prensión con la prótesis y su respectivo control con las señales mioeléctricas. 


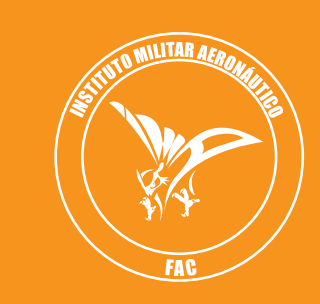

Tecnología

Revista Ciencia y Poder Aéreo. Edición No 6 - Septiembre 2011 ISSN 1909 - 7050

Durante el desarrollo de la tarea de prensión, el sujeto verbaliza las relaciones que observa y descubre que este ejercicio exige la reestructuración y configuración de relaciones ante situaciones de prensión nuevas. Cuando el sujeto emprende el desarrollo de una tarea con la prótesis, esta se simplifica en acciones de Tomar y Poner y debe decidir la secuencia de los pasos básicos. La acción de Tomar, simplemente requiere mover la prótesis para manipular el objeto que previamente se ha decidido, la acción de Poner, solo requiere mover la prótesis para poner el objeto en la posición previamente establecida. Esta relación entre la actividad cognitiva con la respuesta motora evidencia las acciones del pensamiento por medio del movimiento de la prótesis y de esta manera construir un modelo de procesamiento de información a partir de pequeños ejercicios y análisis verbales desarrollados por el sujeto.

\section{Conclusiones}

Se diseñó y construyó una prótesis mecatrónica en aluminio tomando como referencia para sus dimensiones el percentil 50\% de la norma DIN 33 402, que posee 8 grados de libertad (GDL) que le permiten a una persona discapacitada con amputación a nivel inter radial, poder realizar procesos prensiles de precisión tomando señales mioeléctricas del propio cuerpo por medio de una técnica no invasiva de electrodos superficiales, que capturan señales mioeléctricas por medio de un sistema de amplificación de instrumentación médica, el cual permite su reconocimiento y utlilización para la realización de las diferentes tareas de prensión.

El sistema mecatrónico de la prótesis sí cumple con los objetivos de prensión propuestos (cerrar, abrir, cerrar en prensa bi-digital, cerrar en prensa palmar y cerrar en prensa cilíndrica pentadigital).

La gráfica 1 muestra la relación de eficacia de la prótesis por tarea de prensión de acuerdo con los datos obtenidos en relación a los aciertos y errores de las posiciones de prensión para la manipulación de objetos geométricos propuestas por el sujeto en la fase de entrenamiento en el aprendizaje de rutinas motoras de los dos ambientes de aprendizaje.

La prensión de prensa cilíndrica pentadigital es la que mayor eficacia presenta con un $88 \%$, seguida por la posición de cierre completo de la mano con un $85 \%$, la prensa palmar es la que presenta un menor grado de eficacia con un $62 \%$.

Se diseñó e implementó un ambiente de aprendizaje basado en el modelo teórico planteado por Wallon (1970) para que el sujeto que presenta discapacidad por ausencia del miembro superior a la altura del tercio distal del codo, aprenda rutinas motoras en músculos específicos o remanentes para generar movimientos de prensión con la prótesis.

Este modelo se caracteriza por poseer movimientos exógenos, movimientos autónomos y movimientos constructivos aprendidas. El control de la prótesis se realiza por medio de un sistema electrónico de reconocimiento de señales mioeléctricas generadas por el propio sujeto.

Se diseñó e implementó un ambiente de aprendizaje somatognósico basado en el modelo teórico planteado por Ajuariguerra (1976) para que el sujeto que presenta discapacidad por ausencia del miembro superior a la altura del tercio distal del codo, aprenda rutinas motoras en músculos específicos o remanentes para generar movimientos de prensión en la prótesis.

Este modelo se caracteriza por poseer una etapa de exploración por parte del sujeto con la prótesis para descubrirla y reconocerla en sus posibilidades, después una etapa donde el sujeto propone movimientos para la prensión de objetos geométricos presentados de forma aleatoria y por último una etapa donde el sujeto resuelve problemas utilizando las rutinas de prensión aprendidas. El control de estos movimientos es por medio de un sistema electrónico de reconocimiento de señales mioeléctricas del propio sujeto. 

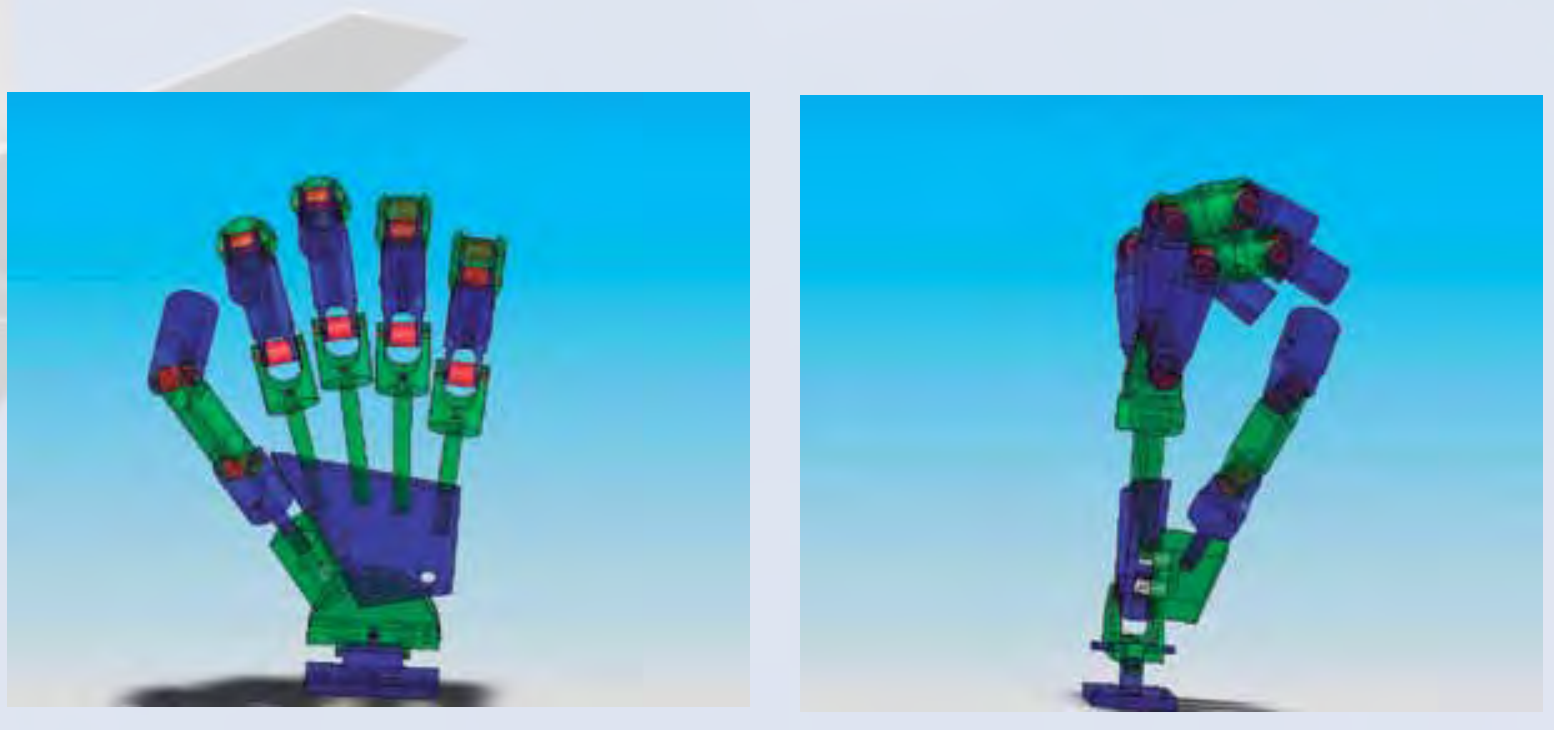

\section{Referencias}

Ausubel, D. (1998). El desarrollo infantil: Aspectos lingüísticos, cognitivos y físicos. Barcelona: Paidós.

Díaz, J. (1999). La enseñanza y aprendizaje de las habilidades y destrezas motrices básicas. Barcelona: Inde publicaciones, p. 234.

Famose, J. (1992). Aprendizaje motor y dificultad de la tarea. Rudik, Psicología. Barcelona: Editorial Paidotribo.

Fonseca, V. D. (2004). Psicomotricidad: Paradigmas del estudio del cuerpo y de la motricidad humana.

Frank, R. W. (2002). La Mano: De cómo su uso configura el cerebro, el lenguaje y la cultura humana.

García Núñez, J. A.,y Berruezo, P. P. (1994). Psicomotricidad y educación infantil y Adelantado. Madrid: CEPE, D.L. ISBN 84-7869-175-8.

Granda J. M. (2001). Manual del aprendizaje y del desarrollo motor. Buenos Aires: Editorial Paidós. (en línea) http://www.estrucplan.com.ar/Producciones/entrega. asp?IdEntrega $=64$

Kurt, M. (1998). Didáctica del movimiento. Berlín: Editorial Volk und Wissen., p. 317
Kurt, M., y Gunter, S. (2004.). Teoría del Movimiento: Motricidad deportiva. Editorial, Stadium S.R.L.

Le Boulch, J. (1978). Hacia una ciencia del movimiento humano. Buenos Aires: Editorial Paidós.

Maisson, J. (2000).Cerebro y Motricidad.

Navarro Adelantado, V. (2002). El afán de jugar: Teoría y práctica de los juegos motores. Editorial INDE.

Oña Sicilia, A. y Martínez Marín, M. E. (1965). Comportamiento motor. Modelos actuales, su aplicación al aprendizaje de habilidades en el aula de educación física.

Rosenzweig, M. (1997). Psicología Fisiológica. McGraw-Hill.

Ruiz Pérez, L. (1982). Desarrollo Motor y Actividad física. Madrid: Gymnos.

(1995). Competencia motriz. Elementos para comprender el aprendizaje motor en Educación Física Escolar. Madrid: Editorial Gymnos.

Wallon, H. (1997). Los estadios en la psicología del niño. Buenos Aires: Ediciones Nueva Visión. 